\title{
Paneth cell dysfunction and the intestinal microbiome in XIAP deficiency
}

\author{
Aline Azabdaftari ${ }^{1}$ and Holm H. Uhlig ${ }^{1-3^{*}}$ \\ 1 Translational Gastroenterology Unit, John Radcliffe Hospital, University of Oxford, \\ Oxford, UK. \\ 2 Department of Paediatrics, University of Oxford, Oxford, UK. \\ 3 NIHR Oxford Biomedical Research Centre, Oxford, UK.
}

*Corresponding author: holm.uhlig@ndm.ox.ac.uk

\begin{abstract}
New data suggest how epithelial dysfunction and intestinal dysbiosis contribute to inflammatory bowel disease in patients and in models of XIAP deficiency (see the related Articles by Strigli et al. and Wahida et al.).
\end{abstract}

Numerous innate and adaptive immune mechanisms ensure that cells of the gastrointestinal tract integrate signals of the billions of bacteria at the mucosal surfaces to provide barrier function and host protective immunity without causing immunopathology. Recent advances in single cell transcriptomic technologies allow mapping of the intestinal cellular landscape unravelling an emerging diversity of new epithelial cell subtypes and activation states within different locations of the gastrointestinal tract (1). These cells adapt to environmental factors (nutrients and microbes), as well as to host factors (endocrine and inflammatory stimuli), both key for maintaining homeostasis and barrier integrity. Paneth cells are integrated mediators of these host-microbe interaction networks. Located at the intestinal crypt base, Paneth cells secrete antimicrobial peptides to regulate microbial colonization and produce growth factors that regulate the epithelial stem cell activity and regeneration. It is increasingly clear that the effects of genetic risk factors that determine the susceptibility to Crohn's disease, such as an ATG16L1 genetic variant, are modulated by environment interactions. Tobacco smoke, a wellestablished risk factor for Crohn's disease, can cause Paneth cell defects in a genetic susceptible host with a common ATG16L1 variant (2). 
Whereas multifactorial inflammatory bowel disease (IBD) is a spectrum of diseases with polygenic contribution, in exceptional cases IBD can be caused by variants in a single gene (for simplicity called monogenic IBD). The X-linked inhibitor of apoptosis encoded by XIAP is a ubiquitin ligase that ubiquitinates RIP kinases and controls signalling of intracellular pathogen recognition receptors as well as cytokine receptors (3). XIAP is expressed in multiple cell types of the innate and adaptive immune system as well as in the epithelial cell compartment. Deficiency of XIAP causes an immune dysregulation disorder with a combination of immunodeficiency and tissue inflammation. This includes susceptibility to infections like Epstein-Barr virus, a macrophage activation syndrome associated with high levels of inflammatory cytokines such as IL-1ß and IL-18 and Crohn's disease like IBD $(3,4)$. Interestingly, IBD is observed only in one third of XIAP deficient patients and the age is variable ranging from infancy to young adulthood. Among the mechanisms that likely contribute to IBD susceptibility in XIAP deficiency is defective NOD2 signalling and RIPK2 dependent defective antimicrobial activity (5). Although changes in the intestinal microbiome (dysbiosis) have been observed in patients with XIAP deficiency (6), it is not clear in humans whether these changes are a cause or consequence of inflammation in particular since many patients receive antibiotic treatments due to systemic infections caused by the primary immunodeficiency, intestinal symptoms and severe Crohn's disease with fistulising phenotype.

In this issue of Science Immunology Strigli et al. and Wahida et al. report on the link between epithelial dysfunction, the microbiome and intestinal inflammation in models of XIAP deficiency and in patients $(7,8)$. As in many other models of IBD, intestinal inflammation in XIAP deficiency depends on the presence of an inflammation supporting microbiota. XIAP deficient mice develop intestinal inflammation under conventional housing conditions associated with an altered composition of the microbiome, whereas under specific pathogenfree conditions spontaneous colitis is not observed $(7,8)$. However, an additional trigger such as a treatment with the chemical dextran sulfate sodium or infection with the facultative pathogenic bacterium Helicobacter hepaticus causes intestinal inflammation with formation of granuloma in the terminal ileum very similar to what is observed in patients with Crohn's disease. In addition to changes in the microbial community structure, an altered bacterial localization within the crypts and close to the epithelium is observed in XIAP deficient mice (8). Intestinal inflammation is associated with a reduced gene expression of antimicrobial peptides and antibactericidals and with the loss of protective microbial species in particular 
Clostridia spp. (7). Strigli et al. and Wahida et al. identify numeric and functional defects in Paneth cells in the intestine of XIAP deficient mice and XIAP deficient patients $(7,8)$. Paneth cells show a reduced response to microbial triggers in XIAP deficient murine epithelium, that is associated with an altered TLR5 and TNF receptor signalling in Paneth cells (7). The Paneth cell loss is mechanistically controlled by TNFR1, RIPK1 and RIPK3 signalling $(7,8)$. These findings altogether suggest a complex mechanism with several amplification loops: defective Paneth cells fail to control the microbiome; dysbiosis causes Paneth cell defects and epithelial barrier breakdown which leads to an increased translocation of bacteria. This causes an inflammatory immune response in a genetic susceptible host, again amplifying TNF dependent Paneth cell death (Figure 1). These studies illustrate that even in a monogenic disorder classified as a primary immunodeficiency, additional features can affect epithelial cell function and Paneth cell activity.

This draws attention to reoccurring pathogenic features such as defective Paneth cell function and defective antimicrobial activity that apply to polygenic and monogenic forms of IBD. A number of puzzling questions remain. Recent data suggests that genetic variants in NOD2 cause an ileocolonic IBD phenotype including strictures through activation of macrophages and fibroblasts via IL-6 cytokine family receptor GP130 dependent mechanisms (9). Given the established role of XIAP within the NOD2-RIPK2 signalling cascade, it is likely that XIAP will mediate similar effects. However, it is surprising that patients with XIAP deficiency instead present with granulomatous colitis and perianal invasive disease as well as macrophage activation defects not typical in IBD patients with heterozygous and biallelic loss of function of NOD2 variants. This indicates a role of XIAP deficiency beyond NOD2 signalling; for instance, additional and potentially synergistic pathogenic mechanisms might be caused by defective NOD1 signalling or non-canonical activities of XIAP.

Whereas medical treatments that target inflammatory cytokines such as IL-1ß and IL-18 have been suggested in XIAP deficiency, and correcting the haematopoietic compartment is an option (3), the question remains which therapeutic strategies can support epithelial function and prevent Paneth cell defects even in the presence of pathogenic XIAP variants. In this context it will be interesting to investigate whether biological therapies that target TNF as standard of care for patients with Crohn's disease work partially by preventing TNF-mediated Paneth cell defects as suggested by animal studies (2). Haematopoietic stem cell transplant (HSCT), the only curative treatment option in XIAP deficiency so far, has a variable outcome, 
and some patients develop severe inflammatory complications after HSCT suggesting an involvement of non-hematopoietic effectors. Recent studies show that HSCT in XIAP deficiency can rescue the IBD phenotype and improve bacterial diversity (6). Future studies will be required to identify whether development of intestinal inflammation or the intestinal complications post-HSCT can be prevented by early resetting of the dysbiosis. This is an important consideration beyond current therapies since future treatment options might include gene therapy where the phagocyte and lymphocyte defects are corrected by reengineered XIAP but epithelial defects remain. Novel therapies that target RIP kinases might have potential in the treatment of intestinal inflammation considering that inhibition of RIPK1 rescued Paneth cell loss in XIAP deficient mice (8). However, further insight into the host protective mechanisms of RIPK1 activity is required since patients with biallelic loss of function variants in RIPK1 present with immunodeficiency as well as tissue inflammation including arthritis and IBD-like intestinal inflammation (10).

Although monogenic IBD suggests a considerably simpler genetic structure compared to polygenic diseases, it doesn't mean that the pathogenesis is simple. The case of XIAP deficiency illustrates the connectivity of signalling pathways and cells within the mucosal barrier function that cannot be illustrated by a single sequential inflammatory pathway nor mapped onto a small group of immune and non-immune cells. Defective Paneth cell function is one overarching integrated feature in subsets of patients with common and rare forms of inflammatory bowel disease modulated by environmental factors. Understanding these defects may allow for more impactful treatments.

\section{Fig. 1. Intestinal inflammation is linked to epithelial barrier function, dysbiosis and dysregulated immune cell activation.}

Under homeostatic conditions, the intestinal epithelial cell barrier allows very few bacteria to translocate through the epithelium, where bacteria are ingested and degraded by phagocytes such as monocytes, macrophages and dendritic cells. This allows host protective immunity and limits inflammatory adaptive immune responses.

XIAP deficiency compromises epithelial barrier function, with Paneth cell loss or dysfunction causing a reduced secretion of antimicrobial peptides. This causes an altered microbial diversity (i.e. loss of protective Clostridia species) and susceptibility to pathogenic bacteria 
(such as Helicobacter hepaticus infection). In XIAP deficiency Paneth cells are activated by TLR5 dependent TNF signalling via TNFR1 which initiates a RIPK1and RIPK3 dependent Paneth cell death. This causes a breakdown of the epithelial barrier allowing more bacteria to penetrate the subepithelial space. Impaired bacterial killing by monocytes and macrophages causes dysregulated inflammatory cytokine secretion (TNF $\alpha$, IL-1 $\beta$, IL-18 and others). A contribution of adaptive immune cells to the pathogenesis in XIAP deficiency is likely but not fully understood.

\section{References and notes}

1. R. Elmentaite, N. Kumasaka, K. Roberts, A. Fleming, E. Dann, H. W. King, V.

Kleshchevnikov, M. Dabrowska, S. Pritchard, L. Bolt, S. F. Vieira, L. Mamanova, N. Huang, F. Perrone, I. G. Kai'En, S. N. Lisgo, M. Katan, S. Leonard, T. R. W. Oliver, C. E. Hook, K. Nayak, L. S. Campos, C. D. Conde, E. Stephenson, J. Engelbert, R. A. Botting, K. Polanski, S. van Dongen, M. Patel, M. D. Morgan, J. C. Marioni, O. A. Bayraktar, K. B. Meyer, X. He, R. A. Barker, H. H. Uhlig, K. T. Mahbubani, K. Saeb-Parsy, M. Zilbauer, M. R. Clatworthy, M. Haniffa, K. R. James, S. A. Teichmann, Cells of the human intestinal tract mapped across space and time. Nature. 597, 250-255 (2021).

2. T.-C. Liu, J. T. Kern, K. L. VanDussen, S. Xiong, G. E. Kaiko, C. B. Wilen, M. W. Rajala, R. Caruso, M. J. Holtzman, F. Gao, D. P. B. McGovern, G. Nunez, R. D. Head, T. S. Stappenbeck, Interaction between smoking and ATG16L1T300A triggers Paneth cell defects in Crohn's disease. J Clin Invest. 128, 5110-5122 (2018).

3. A. C. A. Mudde, C. Booth, R. A. Marsh, Evolution of Our Understanding of XIAP Deficiency. Frontiers Pediatrics. 9, 557 (2021).

4. S. Rigaud, M.-C. Fondanèche, N. Lambert, B. Pasquier, V. Mateo, P. Soulas, L. Galicier, F. L. Deist, F. Rieux-Laucat, P. Revy, A. Fischer, G. de S. Basile, S. Latour, XIAP deficiency in humans causes an X-linked lymphoproliferative syndrome. Nature. 444, 110-114 (2006).

5. T. Schwerd, S. Pandey, H.-T. Yang, K. Bagola, E. Jameson, J. Jung, R. H. Lachmann, N. Shah, S. Y. Patel, C. Booth, H. Runz, G. Düker, R. Bettels, M. Rohrbach, S. Kugathasan, H. Chapel, S. Keshav, A. Elkadri, N. Platt, A. M. Muise, S. Koletzko, R. J. Xavier, T. Marquardt, F. Powrie, J. E. Wraith, M. Gyrd-Hansen, F. M. Platt, H. H. Uhlig, Impaired antibacterial autophagy links granulomatous intestinal inflammation in Niemann-Pick disease type C1 and XIAP deficiency with NOD2 variants in Crohn's disease. Gut. 66, 1060-1073 (2017).

6. S. Ono, K. Takeshita, Y. Kiridoshi, M. Kato, T. Kamiya, A. Hoshino, M. Yanagimachi, K. Arai, I. Takeuchi, N. Toita, T. Imamura, Y. Sasahara, J. Sugita, K. Hamamoto, M. Takeuchi, S. Saito, M. Onuma, H. Tsujimoto, M. Yasui, T. Taga, Y. Arakawa, Y. Mitani, N. Yamamoto, K. Imai, W. Suda, M. Hattori, O. Ohara, T. Morio, K. Honda, H. Kanegane, Hematopoietic cell transplantation rescues inflammatory bowel disease and dysbiosis of gut 
microbiota in XIAP deficiency. J Allergy Clin Immunol Pract (2021), doi:10.1016/j.jaip.2021.05.045.

7. A. Wahida, M. Müller, A. Hiergeist, B. Popper, K. Steiger, C. Branca, M.

Tschurtschenthaler, T. Engleitner, S. Donakonda, J. D. Coninck, R. Öllinger, M. K. Pfautsch, N. Müller, M. Silva, S. Usluer, J. P. Böttcher, N. Pfarr, M. Anton, J. B. Slotta-Huspenina, A. G. Nerlich, T. Madl, M. Basic, A. Bleich, G. Berx, J. Ruland, P. A. Knolle, R. Rad, T. E. Adolph, P. Vandenabeele, H. Kanegane, A. Gessner, P. J. Jost, M. Yabal, XIAP restrains TNF-driven intestinal inflammation and dysbiosis by promoting appropriate immune responses of Paneth and dendritic cells. Sci. Immunol. (2021).

8. A. Strigli, S. Gopalakrishnan, Y. Zeissig, M. Basic, J. Wang, T. Schwerd, S. Doms, K. Peuker, J. Hartwig, J. Harder, P. Hönscheid, P. Arnold, T. Kurth, F. Rost, B.-S. Petersen, M. Forster, A. Franke, J. R. Kelsen, M. Rohlfs, C. Klein, A. M. Muise, N. Warner, R. Nambu, J. Mayerle, H.-P. Török, A. Linkermann, M. H. Muders, G. B. Baretton, J. Hampe, D. E. Aust, J. F. Baines, A. Bleich, S. Zeissig, Deficiency in X-linked inhibitor of apoptosis protein promotes susceptibility to microbial triggers of intestinal inflammation. Sci. Immunol. (2021).

9. S. Nayar, J. K. Morrison, M. Giri, K. Gettler, L. Chuang, L. A. Walker, H. M. Ko, E. Kenigsberg, S. Kugathasan, M. Merad, J. Chu, J. H. Cho, A myeloid-stromal niche and gp130 rescue in NOD2-driven Crohn's disease. Nature. 593, 275-281 (2021).

10. D. Cuchet-Lourenço, D. Eletto, C. Wu, V. Plagnol, O. Papapietro, J. Curtis, L. CeronGutierrez, C. M. Bacon, S. Hackett, B. Alsaleem, M. Maes, M. Gaspar, A. Alisaac, E. Goss, E. AlIdrissi, D. Siegmund, H. Wajant, D. Kumararatne, M. S. AlZahrani, P. D. Arkwright, M. Abinun, R. Doffinger, S. Nejentsev, Biallelic RIPK1 mutations in humans cause severe immunodeficiency, arthritis, and intestinal inflammation. Science. 361, 810-813 (2018).

\section{Acknowledgment}

HUU is supported by the NIHR Biomedical Research Centre Oxford, and The Leona M. and Harry B. Helmsley Charitable Trust.

\section{Competing interests}

HHU has received research support or consultancy fees from Janssen, UCB Pharma, Eli Lilly, BMS/Celgene, MiroBio, OMass, Mestag. AA is funded by an Oxford-BMS fellowship. 